scale motions. This task requires, among other things, research on the synoptic variability of gravity waves. An important step in this direction has recently been taken by Ecklund et al. ${ }^{15}$ who used part of the yet unfinished Poker Flat MST radar in Alaska to demonstrate that gravity wave activity is strongly controlled by propagating planetary waves. Whenever intense baroclinic zones moved across the radar site, the power of the gravity wave spectrum at tropospheric heights increased significantly. Intense baroclinic zones in turn are associated with strong winds and wind shears pointing towards $\mathrm{K}-\mathrm{H}$ instability as a major source mechanism. Figure 2 shows the variation of the square root of the gravity wave spectral power along with the mean vertical wind shear for a period of 34 days indicating a good correlation between both quantities. This strongly suggests that $\mathrm{K}-\mathrm{H}$ instabilities affect the life expectation of synoptic disturbances by continuously borrowing from their energy and momentum budgets.

Future applications of MST radars will be directed towards more intensive studies of sources and sinks of gravity waves and their interaction with large-scale and smallscale atmospheric motions. Preliminary investigations during thunderstorms indicate, for example, that fast-rising air parcels in cumulus clouds can penetrate through the tropopause into the convectively stable stratosphere, thereby exciting a broad spectrum of gravity waves $^{13}$. At heights below $100 \mathrm{~km}$, genesis of turbulence is probably the most important wave sink besides absorption at critical levels. Radar observations show that strong bursts of turbulence arise

\section{Woodman, R.F. \& Guillèn, A. J. atmos. Sci. 31, 493 (1974). \\ . Green, J.L., Warnock, J.M., Winkler, R.H. \& VanZandt, T.E. Geophys. Res. Lett. 2, 19 (1975). \\ 3. Röttger, J., Klostermeyer, J., Czechowsky, P., Rüster, R. \& Schmidi, G. Naturwissenschaften. 65, 285 (1978). \\ 4. Balsley, B.B., Ecklund, W.L., Carter, D.A.\& Johnsion, P.E. Radio Sci. 15, 213 (1980). \\ 5. Gordon, W.E., Bowhill, S.A., Evans, J.V. \& VanZand, T.E. Atmospheric Dynamics in the 1980s, Position Pap. for the 1978 Summer Study Programs of the National Academy of Science Committee on Solar-Terrestrial Research, Rice University, Texas (1978). \\ 6. Rötger, J. \& Schmidt, G. IEEE Trans. Geosci. Electr.} $G E-17,182(1979)$ whenever a gravity wave generates convectively unstable regions ${ }^{14}$. Such bursts are probably identical with clear air turbulence that occasionally threatens aircrafts. In considering the stability of gravity waves we arrive at the problem of parametric instabilities. Waves with finite amplitudes so small that shear or convective instabilities do not occur should enhance a spectrum of disturbance waves by their rocking motions (like the excitation of a swing by a child). This is one of a variety of nonlinear gravity wave processes awaiting their discovery by MST radar experiments.

7. Rastogi, P.K. \& Bowhill, S.A. J. atmos. terr. Phys. 38, 51 (1976)

8. Rüster, R., Rötıger, J. \& Woodman, R.F. Geophys. Res. Lett. 5, 555 (1978).

9. Fukao, S., et al. J. geophys. Res. 84, 4379 (1979).

10. Klostermeyer, J. \& Liu, C.H. Geophys. Res. Lett. 6, 507 (1978).

11. VanZandt, T.E., Green, J.L., Clark, W.L. \& Grant, J.R. Geophys. Res. Lett. 6, 429 (1979).

12. Klostermeyer, J. \& Rüster, R.J. Geophys. Res. 85, 2841 (1980).

13. Röltger, J. Pageoph 118, 494 (1980)

14. Klostermeyer, J. \& Ruster, R.J. Geophys, Res. in the press).

15. Eckland, W. L., Gage, K.S. \& Riddle, A.C. Geophys. Res. Lett. 8, 285 (1981).

\title{
X-ray laser obtained by pumping with a nuclear bomb?
}

\section{from Peter Knight}

RUMOURS have been buzzing around the laser community for some months that an $\mathrm{X}$-ray laser has been made to work by a group led by George Chapline of Lawrence Livermore National Laboratory. Initial reports in the February 23 issue of $A$ viation Week and Space Technology (stressing military applications) have been followed by notes in the April and May issues of Laser Focus that the Livermore group tested a device pumped by a small nuclear bomb at the DoE Nevada Test Site and produced a pulse of several hundred terawat ts of X rays at 1.4 nanometres. So far no official comment or confirmation has been made by the Livermore. As the correspondent of Laser Focus points out, 1.4 nanometres corresponds to the $\mathrm{K} \alpha$ line of neon. Early reports that nanosecond pulses were produced seem unlikely since such a device would probably operate as a singlepass self-terminating amplified spontaneous emission system limited at least by the picosecond upper state lifetimes. Picosecond or femtosecond pulse lengths would be more reasonable. The rumoured experiment has attracted much interest and a full account is eagerly awaited. Past efforts in this field have not survived careful scrutiny. Since a nuclear bomb is necessary in the present case, it seems difficult to check by independent experiment.

It is very difficult to create the right conditions for X-ray laser action. No mirrors are envisaged and single-pass gain must be high enough that spontaneous fluorescence is efficiently amplified by stimulated emission during its transit down a rod-shaped inverted medium. A gain of about $100 \mathrm{~dB}$ is required according to $\mathrm{G}$. Chapline and L. Wood (Physics Today June 1975, p.40) and this requires inversion densities $N^{*}>(\Delta v / L) \times 10^{18} \mathrm{~cm}^{-3}$ where $\Delta v$ is the bandwidth in electron volts and $L$ is the length of the inverted medium in centimetres. 'Cold matter' has an Auger bandwidth $\Delta v \sim 1 \mathrm{eV}$ and enormous pump intensities are required to reach such large values of $N^{*}$. In a plasma, $\Delta v$ is governed by Stark widths and then $N^{*}>5 \times 10^{19} / L$ $\mathrm{cm}^{-3}$ which requires fractional inversion $N^{*} / N \sim 10^{-3}$. If pump intensities are such that $N^{*} / N<10^{-3}$, then one way out is to use material of more than solid density obtained through some compression scheme. It is interesting to note in this context that the Livermore experiment was supposed to be pumped by a nuclear bomb.

Many schemes for producing X-ray population inversion have been studied. Most use very high-power laser excitation of plasma targets. Promising results using this route have been reported by workers at the University of Hull (D. Jacoby et al. Opt. Commun. 37; 193, 1981) with the Balmer $\alpha$ line of Carbon VI at $182 \AA$. An early proposal due to $\mathrm{M}$. Duguay and $\mathrm{P}$.

Peter Knight is in the Optics Section of the Blackett Laboratory, Imperial College, London.
Rentzepis (Appl. Phys. Lett. 10; 350, 1967) suggested exploiting the frequency dependence of photoionization crosssections to produce inversion. Tightly bound electrons are actually more easily photoionized by $\mathrm{X}$ rays than weakly bound electrons, so that an incident high-intensity flash of $\mathrm{X}$ rays could produce population inversion by leaving ions in excited states. Conventional X-ray sources are not bright enough to achieve the required gain. It is possible that nuclear explosions can produce the required rapid inversion and one might guess that this was used in the Livermore experiment.

The properties of coherent X-ray sources are so valuable that great efforts are being made to construct a useful X-ray laser device. If one were available, phasecontrast microscopy would make atomicscale resolution of the dynamics of biological systems in situ possible, could solve the phase problem in X-ray crystallography and lead to the study of atomic dynamics with $\sim 10^{-15}$ second resolution. The nuclear bomb-pumped single-shot $X$-ray laser would be of value in scientific diagnostics but would not seem to be a route to a useful device. Nevertheless, it does represent an invigorating advance which might help in obtaining funds to continue the expensive investigation of potential X-ray laser systems. We will have to wait for more details than the leaked rumours before a proper assessment can be made. 